\title{
Renal replacement therapy in cancer patients with acute kidney injury (Review)
}

\author{
MIRCEA LUPUŞORU ${ }^{1}$, GABRIELA LUPUŞORU ${ }^{2,3}$, IOANA AILINCĂI ${ }^{2}$, GEORGIANA FRĂȚILĂ $\breve{̆}^{2}$, \\ ANDREEA ANDRONESI ${ }^{2,3}$, ELENA MICU ${ }^{2,3}$, MIHAELA BANU $^{4}$, RADU COSTEA $^{5}$ and GENER ISMAIL ${ }^{1,2}$ \\ ${ }^{1}$ Department of Physiology 1, 'Carol Davila' University of Medicine and Pharmacy, 020021 Bucharest; \\ ${ }^{2}$ Department of Nephrology, 'Fundeni' Clinical Institute, 022328 Bucharest; Departments of ${ }^{3}$ Uronephrology, \\ ${ }^{4}$ Morphology and ${ }^{5}$ General Surgery, 'Carol Davila' University of Medicine and Pharmacy, 020021 Bucharest, Romania
}

Received April 20,2021; Accepted May 20, 2021

DOI: $10.3892 / \mathrm{etm} .2021 .10296$

\begin{abstract}
Cancer patients are at high risk for developing acute kidney injury (AKI), which is associated with increased morbidity and mortality in these patients. Despite the progress made in understanding the pathogenic mechanisms and etiology of AKI in these patients, the main prevention consists of avoiding medication and nephrotoxic agents such as non-steroidal anti-inflammatory drugs, contrast agents used in medical imaging and modulation of chemotherapy regimens; when prophylactic measures are overcome and renal impairment becomes unresponsive to treatment, renal replacement therapy (RRT) is required. There are several methods of RRT that can be utilized for patients with malignancies and acute renal impairment; the choice of treatment being based on the patient characteristics. The aim of this article is to review the literature data regarding the epidemiology and management of AKI in cancer patients, the extracorporeal techniques used, choice of the appropriate therapy and the optimal time of initiation, and also the dose-prognosis relationship.
\end{abstract}

\section{Contents}

1. Introduction

2. Definition of AKI

3. Epidemiology of AKI in cancer patients

4. Indications for kidney replacement therapy in AKI

5. Renal replacement therapies

6. Choosing the right therapy (IRRT/CRRT), optimal time of initiation and dose/prognosis relationship

7. Extracorporeal blood purification techniques in oncology

8. Conclusions

Correspondence to: Dr Gabriela Lupuşoru, Department of Uronephrology, 'Carol Davila' University of Medicine and Pharmacy, 37 Dionisie Lupu Street, 020021 Bucharest, Romania E-mail: gabrielatopor@yahoo.com

Key words: cancer, acute kidney injury, intermittent renal replacement therapy, continuous renal replacement therapy, hybrid therapy

\section{Introduction}

Patients presenting with malignancies have a high risk of developing acute kidney injury (AKI) secondary to receiving chemotherapy, exposure to contrast agents used in medical imaging, radiation therapy, tumor lysis syndrome, hypotension or caused by the direct effects of the malignancy. $\mathrm{AKI}$ is a frequent complication in cancer patients and is associated with increased morbidity and mortality. Because a significant percentage of cancer patients with AKI eventually require renal replacement therapy (RRT), it is important to know which method of RRT is appropriate depending on the context (intermittent or continuous hemodialysis or plasmapheresis), what is the optimal time of initiation and whether or not it can improve outcomes in terms of recovery of kidney function and increasing survival. There are few data in the literature that provide conclusive information regarding the appropriate time of dialysis initiation and discontinuation and on the prognosis of these patients, especially for patients with multiple system organ failure or with uncontrolled cancer. Thus, the benefit of RRT must be evaluated according to the prognosis of the patient, by a multidisciplinary team including a nephrologist, oncologist and intensivist.

AKI in patients with malignancies can be caused by: i) The direct effects of the malignancy (lymphoma infiltration of the kidney), ii) Hematopoietic stem cell transplantation, iii) Chemotherapy-related injury (tumor lysis syndrome), iv) Drug-associated nephrotoxicity (acute tubular injury), v) Obstructive nephropathy due to urothelial or retroperitoneal cancers, vi) Nephrectomy (in the case of kidney cancer) which increases the risk of renal failure and the need for dialysis (1-3), vii) Volume depletion secondary to vomiting as a side effect of cancer treatment, viii) Paraneoplastic syndromes which can compromise the renal function: Syndrome of inappropriate secretion of antidiuretic hormone (SIADH), hypercalcemia (4), and tumor lysis syndrome that may require dialysis in extreme situations.

Risk factors associated with the development of AKI in cancer patients include: Female sex, older age, diabetes mellitus, the presence of underlying chronic kidney disease, hypotension and inadequate renal perfusion (5). 


\section{Definition of AKI}

In the last few decades, different definitions of AKI have been applied, the most used being those taking into consideration the urine output and serum creatinine. The multitude of definitions has made it difficult to compare data from different studies regarding AKI. The KDIGO workgroup on AKI standardized these variations into a single definition and a staging system that is being used today (6) (Table I).

\section{Epidemiology of AKI in cancer patients}

A Danish population-based cohort study reported the incidence of AKI in cancer patients followed up for more than a period of 7 years (7). Of 1.2 million individuals, 44,116 developed a malignancy. The risk of AKI was 17.5\% during the first year after cancer diagnosis and the overall 5-year risk of AKI was $27.0 \%$. The highest incidence rates were in patients with cancer localized in the kidney (44\%), biliary tract, liver, pancreas, and in patients with multiple myeloma. Among patients with renal impairment, 7\% had severe AKI and 5\% required RRT.

High AKI incidence rates are also noted in men with prostate cancer (29\%) and urinary bladder cancer (36.3\%), but also in women with ovarian $(36.2 \%)$ or uterus/cervical cancers (26.7/28.6\%) (7). Considering that prostate cancer is the second most commonly diagnosed cancer in men in Europe, accounting for $10-15 \%$ of all diagnosed cancers (8-10) and the most frequent neoplastic pathology in men of age over 60 years (11), and also that uterus and cervical cancers are a major health issue for European women, with the biggest morbidity and mortality rates among Romanians (12), we need to provide access to optimal treatment and the current health programs must be adapted for a better prevention of neoplastic disease and its complications-AKI and the need for renal replacement in these patients, with the most appropriate method of dialysis and the optimal duration and dose of therapy.

\section{Indications for kidney replacement therapy in AKI}

The standard indications for RRT initiation in the acute setting are well known: Fluid overload resistant to diuretic therapy, refractory acid-base and hydroelectrolytic disorders, uremic pericarditis, pleuritis, encephalopathy or progressive neuropathy (Table II) $(13,14)$. It remains uncertain whether earlier initiation of such therapy can improve outcomes in terms of the recovery of kidney function and decreasing mortality in cancer patients.

\section{Renal replacement therapies}

Dialysis principles. There is no ideal method of RRT. The ideal method of kidney replacement therapy should allow control of the intravascular and extravascular volume, correction of acid-base imbalances, correction of uremia, an effective removal of toxins, while also promoting recovery of renal function, increasing survival without complications.

Blood purification through the artificial kidney is governed by physical forces: Diffusion and convection (ultrafiltration) exerted on the surface of the dialyzer membrane, a semipermeable membrane through which the patient's blood is brought into contact with the dialysis fluid. There are two methods used to correct the acid-base and electrolyte imbalances and remove toxins and excess fluid: Dialysis (method that uses diffusion) and ultrafiltration (method that uses hemofiltration) (15).

In the case of continuous or intermittent dialysis, the mechanism of solute transport is diffusion-the passage of solvents from one compartment to another through a semipermeable membrane, according to a concentration gradient and depending on time, molecular mass of the substances passing through it and membrane pore size. By diffusion we achieve a very good clearance for small molecules, below $500 \mathrm{Da}$ : Urea, creatinine, ions. The transport of solutes between the two compartments is also the result of the Brownian motion. Thus, larger molecules, such as mediators of inflammation [interleukin (IL)-6, tumor necrosis factor (TNF) $\alpha$ ] that are found in high amounts in critically ill septic patients or in patients in shock, will collide with the semipermeable membrane less often due to their slower movement in the liquid medium, which will cause a deficient clearance of these molecules (15).

When using convection, solutes pass from one compartment to another through the semipermeable membrane according to a pressure gradient created by a pump. Negative pressure is being applied in the dialysate compartment and water is being dragged through the membrane accompanied by solutes. This is the mechanism used in hemofiltration. The clearance of a molecule is the product between the ultrafiltration rate and the selectivity (separation factor); thus, to increase the clearance when the selectivity is low, one must increase the ultrafiltration rate (15).

Modalities of RRT in patients with malignancies and AKI. There are 3 methods of RRT used in patients with malignancies and acute renal impairment (Table III). These include: i) Continuous renal replacement therapy (CRRT): Continuous veno-venous hemofiltration $(\mathrm{CVVH})$, continuous veno-venous hemodialysis (CVVHD), continuous veno-venous hemodiafiltration (CVVHDF), slow continuous ultrafiltration (SCUF); ii) Intermittent renal replacement therapy (IRRT): Intermittent hemodialysis (IHD), intermittent hemodiafiltration (IHDF), intermittent isolated ultrafiltration (IUF); and iii) Hybrid therapy: Sustained (slow) low efficiency daily dialysis (SLEDD/SLEDD-F), prolonged intermittent renal replacement therapy (PIRRT) $(16,17)$.

Hybrid therapies use the standard dialyzer, the difference being time. The hemodialysis session last for $4 \mathrm{~h}$, while SLEDD lasts for $12 \mathrm{~h}$. The continuous renal replacement therapies take place in intensive care units, using special dialysis machines (16).

Intermittent therapies. IRRT are used in hemodynamically stable patients. They are performed on regular dialysis machines, are relatively cheaper and have a series of advantages which include the possibility of establishing a more flexible schedule of sessions-which offers advantage over patient transport and administration of dialyzed drugs, quick correction of acid-base and electrolyte imbalances and have a lower risk of bleeding (16).

They also have certain disadvantages, the best known of them being intradialytic hypotension and cerebral edema (18). 
Table I. Definition and staging of AKI (6).

\begin{tabular}{lll}
\hline Stage & \multicolumn{1}{c}{ Serum creatinine } & \multicolumn{1}{c}{ Urine output } \\
\hline 1 & $>1.5-1.9$ times baseline or $>0.3 \mathrm{mg} / \mathrm{dl}$ increase & $<0.5 \mathrm{ml} / \mathrm{kg} / \mathrm{h} \mathrm{for} 6-12 \mathrm{~h}$ \\
2 & $\geq 2-2.9$ times baseline & $<0.5 \mathrm{ml} / \mathrm{kg} / \mathrm{h}$ for $\geq 2 \mathrm{~h}$ \\
3 & $>3$ times baseline or $>4 \mathrm{mg} / \mathrm{dl}$ increase or Renal replacement & $<0.3 \mathrm{ml} / \mathrm{kg} / \mathrm{h}$ for $>24 \mathrm{~h}$ or Anuria for $>12 \mathrm{~h}$ \\
& therapy initiation or In patients $<18$ years, a decrease in & \\
& eGFR to $<35 \mathrm{ml} / \mathrm{min}$ per $1.73 \mathrm{~m}^{2}$ & \\
\hline
\end{tabular}

AKI, acute kidney injury; eGFR, estimated glomerular filtration rate.

Table II. Indications for initiation of RRT (adapted from ref. 13,14).

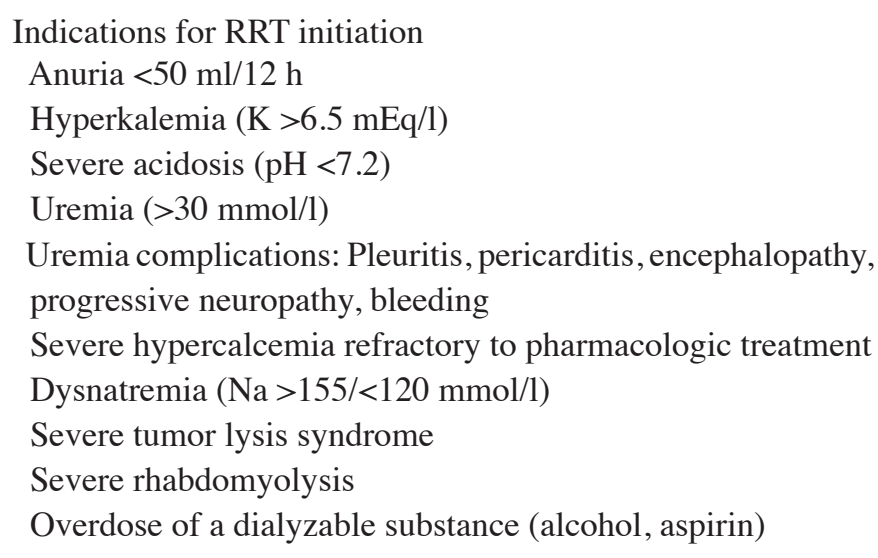

RRT, renal replacement therapy.

Intradialytic hypotension occurs by intravascular volume reduction through the ultrafiltration process and can cause coronary ischemia, intestinal ischemia and increases the renal injury. Risk factors for intradialytic hypotension include: Left ventricular hypertrophy with systolic or diastolic dysfunction, valvulopathies, pericardial involvement, uremic neuropathy, severe anemia, predialysis systolic blood pressure less than $100 \mathrm{mmHg}$, poor nutritional status, high volume ultrafiltration and age over 65 (18).

Cerebral edema, causing a range of neurologic symptoms that form the dialysis disequilibrium syndrome, occurs particularly when a patient is first started on dialysis. It is caused by the rapid reduction during hemodialysis sessions in blood levels of osmotically active substances, which makes the plasma more hypotonic compared to the brain tissue, favoring the passage of water from the blood to the brain and the appearance of symptoms: Nausea, vomiting, headache, and dizziness $(18,19)$. Another mechanism by which cerebral edema occurs is related to the decrease in urea transporters and the overexpression of aquaporins in the brain (19). Risk factors for cerebral edema in the first dialysis sessions are: BUN >175 mg/dl, rapid decrease in urea level, pre-existing neurological disorders, hyponatremia and liver diseases (18-20).

Continuous therapies. CRRT is recommended in critically ill patients: Hemodynamically unstable, septic, shock patients and mechanically ventilated patients (Table IV) (21). The sessions take place in intensive care units, using special dialysis machines. Although the costs are higher, they offer a series of advantages. The advantages include: A slower decrease in intravascular volume and slower solute clearance which improves hemodynamic stability, thus favoring recovery of renal function, and also ensures a better and more predictable control of the blood parameters and volume, a more stable intracranial pressure and finally ensures a better clearance of cytokines $(16,21)$.

The disadvantages of continuous therapies include: Prolonged patient immobilization and transport problems, increased costs of dialysis fluids and supplies, increased risk of coagulation of dialysis circuits and the use of high doses of anticoagulants-the latter increasing the risk of thrombocytopenia and bleeding, due to prolonged exposure to heparin $(16,18,21)$.

Hybrid therapies. Hybrid therapies (SLEDD, SLEDD-F and PIRRT) combine the advantages of continuous and intermittent therapies. These include: Good clearance for small molecules, improved hemodynamic stability because of slower ultrafiltration, need of lower doses of anticoagulants, lower costs because the sessions can take place on standard dialysis machines $(22,23)$. The major advantage is the flexibility in terms of session duration and its intensity (the ultrafiltration rate can be high, but it can also be adjusted according to the patient's needs). A series of studies that have compared SLEDD to CRRT did not find significant differences between the two methods regarding the hemodynamic parameters measured (mean blood pressure, systemic vascular resistance and LV ejection fraction) (24-26). In addition, the clearance for urea and creatinine was similar (16).

Peritoneal dialysis in cancer patients with AKI. Peritoneal dialysis is being used as RRT in AKI patients only under very specific conditions. This can be useful in hemodynamically unstable patients, in those with high risk of bleeding or with fragility syndrome, but it is less efficient than blood purification techniques, in regards to solute clearance and excess fluid removal $(16,21,27)$.

\section{Choosing the appropriate therapy (IRRT/CRRT), optimal time of initiation and dose/prognosis relationship}

Although there are arguments in favor of higher doses of therapy and better prognosis $(28,29)$, there is still not enough 
Table III. Comparison of the different methods of renal replacement therapy.

\begin{tabular}{lccc}
\hline & Continuous therapies & Intermittent therapies & Hybrid therapies \\
\hline Time (h/day) & 24 & 4 & $8-12$ \\
Blood flow rate (ml/min) & $15-300$ & $300-400$ & $150-300$ \\
Dialysate flow rate (ml/min) & $30-60$ & $600-800$ & 100 \\
Replacement fluid flow rate (ml/min) & $30-60$ & - & 100 \\
Dialysis & Yes & Yes & Yes \\
Hemofiltration & Yes & No & Yes \\
Efficiency & Low-Moderate & High & Moderate \\
Hemodynamic stability & High & Low & High \\
Cost & $\uparrow \uparrow \uparrow$ & $\uparrow$ & $\uparrow \uparrow$ \\
\hline
\end{tabular}

Table IV. Indications for CRRT and SLEDD.

Indications for CRRT and SLEDD

Shock:
Cardiac SOFA score $>2$
Intra-aortic balloon pump
Extracorporeal membrane oxygenator (ECMO)
Cerebral edema
Hepatic failure
Refractory hypervolemia
Rhabdomyolysis
Tumor lysis syndrome
Severe hypercatabolism
Hyperammonemia

CRRT, continuous renal replacement therapy; SLEDD, sustained low efficiency daily dialysis; SOFA, sequential organ failure assessment.

evidence for the superiority of one therapy over another (30). There are large variations in practice and the subject remains open and intensely debated.

Ronco et al (29) published in 2000 a prospective, randomized study of 425 patients with AKI who were treated with CVVH. They were divided into 3 groups according to the ultrafiltration volumes $(\mathrm{ml} / \mathrm{bw} / \mathrm{h})$ and followed the survival rate. The group with the lowest dose of ultrafiltration had the lowest survival. He concluded that an increase in the rate of ultrafiltration improved survival significantly and recommend that ultrafiltration should be prescribed according to patient's bodyweight (bw).

Currently there are contradictory data regarding the relationship between the type of chosen therapy (IRRT or CRRT) and prognosis. Kellum et al (31) published the results of a meta-analysis of 13 studies comparing the effects of intermittent vs. continuous therapy. The primary endpoint was in-hospital mortality. He found no significant differences between the two methods. There were a few studies that compared groups of equal severity of illness at baseline (time of enrollment) and adjusting for study quality and severity of illness, mortality was lower in patients treated with CRRT (31).
Later, Tonelli et al published a meta-analysis of 6 trials, which showed no difference between the two types of therapies (continuous and intermittent) in terms of mortality (32). Of these, only 4 studies (33-36) had data on improving renal function and their analysis showed no significant differences between the two methods.

Another important therapeutical aspect in patients with AKI is the decision concerning the timing of initiating RRT, respectively the effects of early dialysis on survival, recovery of renal function and the number of days spent in intensive care unit. Early initiation of renal replacement therapy may have some advantages in achieving more rapidly a state of euvolemia, electrolyte and acid-base rebalancing and removal of proinflammatory and other toxins from circulation (21). On the other hand, it also has some side effects which include catheter-related infections, hypotensive episodes, and bleeding (21). There were two major trials published in 2016 that investigated whether early renal replacement therapy decreases mortality in critically ill patients with AKI (37). The Early versus Late Initiation of Renal Replacement Therapy in Critically Ill Patients with AKI (ELAIN) trial (38) and The Artificial Kidney Initiation in Kidney Injury (AKIKI) trial (39) are two studies that have brought contradictory results; the former showed that early initiation of RRT (within $8 \mathrm{~h}$ of diagnosis of KDIGO stage 2) significantly reduced 90-day mortality compared with delayed initiation of RRT (within $12 \mathrm{~h}$ of stage $3 \mathrm{AKI}$ ). AKIKI showed no significant difference in 60-day mortality between early and delayed RRT.

Patients should be treated individually based on their characteristics and physician's experience (Table V) (17). Continuous therapy is the best method in hemodynamically unstable patients on at least two vasopressors or respiratory support, those with cerebral edema and craniocerebral trauma and those with severe sepsis $(6,16,21,40)$. Hybrid therapies are indicated rather as transitional therapies to intermittent therapies in patients with progressively reduced doses of vasopressors, when mechanically ventilated patients are extubated or for critically ill patients, hemodynamically unstable and at high risk of bleeding (after surgery or anticoagulant therapy) $(6,16,21)$. Intermittent therapies in AKI should be reserved for life-threatening conditions that require rapid correction (eg severe hyperkalemia) $(6,16,21)$. A special situation is tumor lysis syndrome and rhabdomyolysis which can be treated by IRRT/CRRT combinations $(21,41)$. 
Table V. Choice of renal replacement therapy according to the associated clinical conditions (modified from ref. 17).

\begin{tabular}{lcccc}
\hline & Life-threatening conditions & Hypervolemia & Hemodynamic instability & Cerebral edema \\
\hline First option & IRRT & CRRT/SLEDD/PIRRT & CRRT/SLEDD/PIRRT & CRRT/PD \\
Second option & PIRRT & IRRT & PD & PIRRT \\
Third option & CRRT & PD & IRRT & IRRT \\
Fourth option & PD & - & - & - \\
\hline
\end{tabular}

IRRT, intermittent renal replacement therapy; CRRT, continuous renal replacement therapy; SLEDD, sustained low efficiency daily dialysis; PIRRT, prolonged intermittent renal replacement therapy; PD, peritoneal dialysis.

\section{Extracorporeal blood purification techniques in oncology}

Plasmapheresis is an extracorporeal procedure used in oncology. This is a process in which the liquid part of the blood, or plasma, is separated from the blood cells. Typically, the plasma is replaced with another solution such as fresh frozen plasma or 5\% albumin solution. Plasmapheresis can be intermittent or continuous; there are 'high-volume' or 'ultrahigh-volume' hemofiltration therapies (42).

Plasmapheresis is used in the oncology field for paraneoplastic syndromes with neurological manifestations, Eaton Lambert myasthenic syndrome, paraproteinemias, myelomas, peripheral neuropathies related to paraproteinemias, cytokine release syndrome from sepsis (42).

Patients diagnosed with multiple myeloma usually present to the nephrologist with myelomatous nephropathy, amyloid infiltration of the kidney, or direct tubular light chain toxicity. These patients have a much lower survival rate at 1 year compared to those with normal kidney function. Rapid reduction of light chains is the most important step in the treatment. Early reduction in these chains is associated with an increased rate of renal function recovery (43-45).

In addition to general measures, chemotherapy and stem cell transplantation, recently there is a special interest in extracorporeal purification techniques such as dialysis or plasmapheresis regarding the fact that the renal distress is directly consistent with the serum and urinary level of these light chains $(44,46)$.

In 2011 Hutchison et al (44) published a study of 39 patients from 2 large university centers (Birmingham and Rochester) with histopathological diagnosis of myelomatous nephropathy and AKI, who received either chemotherapy and extensive hemodialysis with protein-permeable dialysis (HF-HD) or chemotherapy and plasmapheresis. The results emphasized that a $60 \%$ reduction in light chains by day 21 of diagnosis was associated with recovery of renal function in $80 \%$ of cases. Thus, there is an increased interest in the use of extracorporeal therapies for rapidly decreasing the serum level of light chains. Only one randomized trial using plasmapheresis for myelomatous nephropathy has been reported. Clark et al (45) published a study of 97 patients with multiple myeloma and presumed myelomatous nephropathy, who were randomly assigned to conventional therapy plus 5 to 7 plasma exchanges of $50 \mathrm{ml}$ per $\mathrm{kg}$ of body weight of $5 \%$ human serum albumin for 10 days or conventional therapy alone. The investigators found no evidence that the use of plasmapheresis improved the survival rate and recovery of renal function. Chemotherapy with melphalan, prednisone and cyclophosphamide was the standard of care in these patients, but the use of new nongenotoxic chemotherapy (bortezomib, thalidomide and lenalidomide) increased interest in extracorporeal treatment of light chains, especially by high cut-off hemodialysis (HCO-HD), which uses high cut-off (HCO) membranes that enables the removal of large molecule, up to $60 \mathrm{kDa}$. However, these membranes allow the passage of plasma proteins, such as albumin, an unwanted loss. These membranes allow for the removal of higher-molecular-weight molecules, such as mediators of sepsis/inflammation or rhabdomyolysis or the removal of nephrotoxic light chains of immunoglobulins, but they have the disadvantage of losing albumin so they are used only for a limited number of sessions (46).

All this information leads to a complex, combined treatment of chemotherapy and hemodialysis.

In the largest study of patients with multiple myeloma and AKI requiring hemodialysis, conducted by Hutchison et al (47), 67 patients from several countries were treated with HCO-HD, most of them being treated also with bortezomib-based chemotherapy or thalidomide. A total of $63 \%$ of patients recovered their renal function. Predictors associated with renal function recovery were the reduction of light chains by day 12 and 21 of treatment and the time until the initiation of hemodialysis. Unfortunately, patients had high cut-off hemodialysis (HCO-HD) together with chemotherapy, while the study had no control group for comparison. Even though the results were promising in terms of reducing light chains, they did not answer the fundamental question of whether hemodialysis with $\mathrm{HCO}$ membranes has benefits in addition to bortezomib-based chemotherapy. This requires randomized trials and there are no prospective randomized controlled group studies published. There are two major trials, a British trial (EuLITE) and a French trial (MYRE), whose results are somewhat contradictory. Both studies enrolled patients with myelomatous nephropathy and AKI and tested the effect of lowering the serum level of light chains on the recovery of renal function. Patients received chemotherapy and conventional hemodialysis or chemotherapy and HCO-HD; at 3 months of treatment there were no significant differences between the two groups of patients regarding independence from dialysis $(48,49)$. However, the MYRE study showed differences at 6 and 12 months (50) in favor of HCO-HD in recovering renal function, but the group of patients was not large enough. 


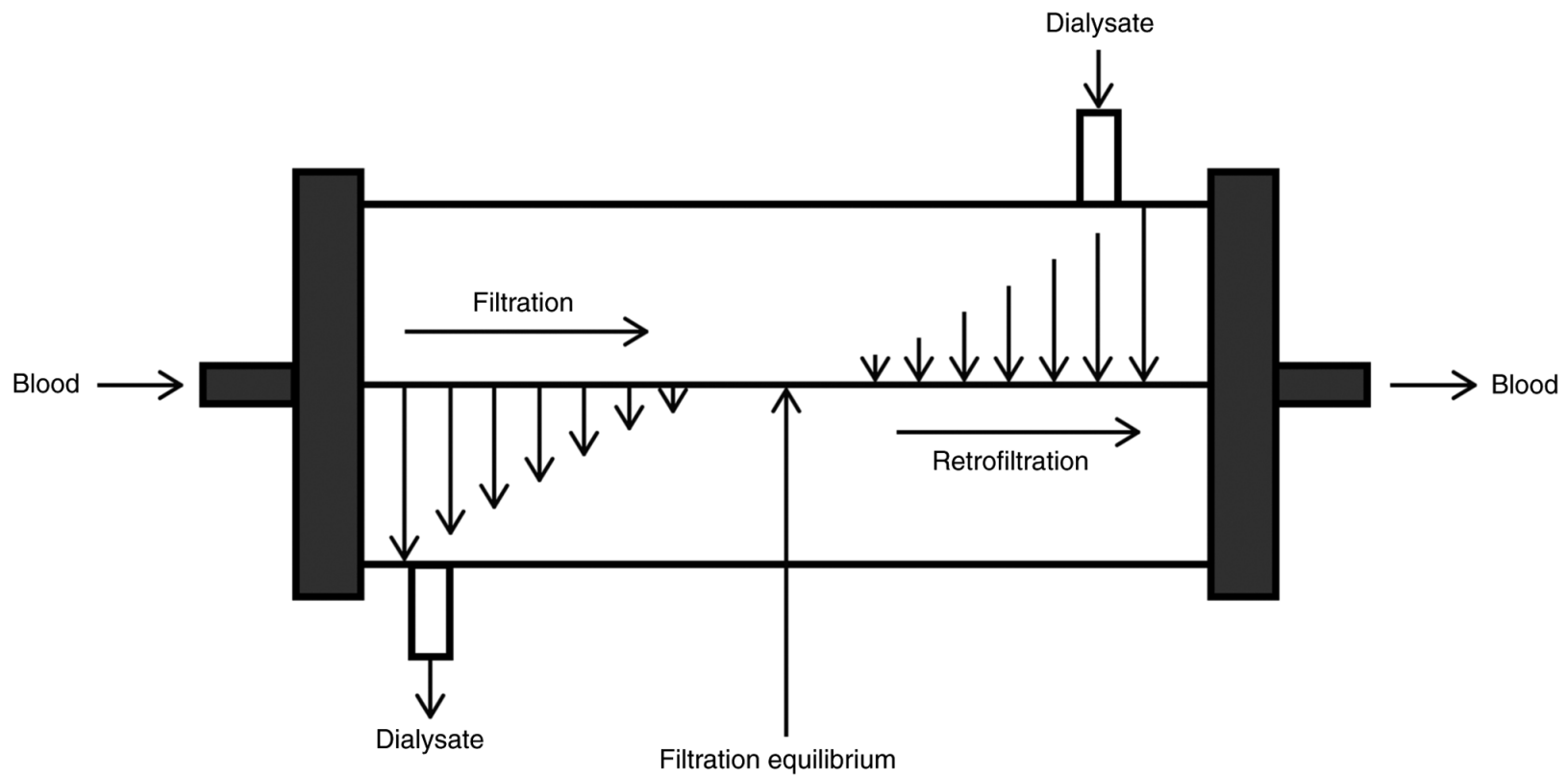

Figure 1. Schematic representation of the MCO hemodialyzer. MCO, medium cut-off.

HCO-HD may not yet be recommended as a routine treatment for these patients considering the lack of large-scale studies.

We still need to deal with the high mortality rate and high morbidity among these patient and new therapeutic solutions are needed despite the progress in the development of hemodialysis regarding survival and quality of life.

Online hemodiafiltration is increasingly used. This method uses high-flow membranes and combines diffusion with convection, with large volumes of ultrafiltration for medium-high molecular-weight molecules (since their purification is dependent on a large convection volume). Thus, a study was published in 2011 (51) comparing the efficacy of online hemodiafiltration with high-flow hemodialysis in patients with multiple myeloma and AKI. The study included 27 patients and showed a higher extraction capacity by hemodiafiltration vs. hemodialysis for both $\mathrm{K}$ and $\lambda$ light chains, although the clearance capacity increased proportionally to the volume of substitution.

Online hemodiafiltration shows good results, but it is still suboptimal and unsatisfactory, with high mortality and cardiovascular morbidity, so that new therapeutic strategies are needed, one of them being extended hemodialysis. This is a process by which diffusion and convection are combined inside a special dialyzer, equipped with a medium cut-off (MCO) membrane. These recently produced MCO membranes with intermediate porosity (between $\mathrm{HF}$ and $\mathrm{HCO}$ ) have certain favorable characteristics such as higher permeability for medium molecules and much lower albumin loss compared to HCO membranes. MCO membrane filtration resembles quite well that of the normal kidney (52-54).

Extended hemodialysis (HDx) is the latest advancement in efficiency and simplification. In HDx the convective transport required to remove medium to large MW solutes is the result of a complex mechanism hidden inside the MCO dialyzer membrane. Manufacturers have reduced the thickness of the semipermeable membrane and the fiber inner diameter, which improved the membrane's permeability and efficiency and solute transport (larger number of fibers per dialyzer making it more compact) (53-56). Reducing the fiber inner diameter increases the wall shear rate with a cleaning effect at the blood membrane interface, which improves the solute transport (53-56). The combination of hydraulic permeability and geometric structure of the fiber increases the process of internal filtration and back filtration. Thus, this mechanism allows a large volume of convection inside the dialyzer, where the filtration takes place in the proximal part and back filtration compensates for the excessive ultrafiltration rate in the distal part (Fig. 1) $(53,55,56)$.

Randomized trials are still needed for definitive conclusion. Given the high cost of MCO filters, online hemodiafiltration is reserved for a limited number of sessions for certain types of patients, such as those with multiple myeloma.

\section{Conclusions}

Acute kidney injury is a common complication among patients with a malignancy of various types, which may require renal replacement therapy. The adequate management of this special group of patients requires the establishment of the most appropriate type of therapy, timing of initiation, the optimal duration and dose of therapy, because all of these aspects influence the recovery of renal function, quality of life and mortality rate of the patients. While waiting for large randomized trials to be published, we have to focus on personalized therapy based on clinical and laboratory characteristics, patient's decision and experience of the nephrologist-oncologist-intensive care team.

\section{Acknowledgements}

Not applicable.

\section{Funding}

No funding was received. 


\section{Availability of data and materials}

All information is documented by relevant references.

\section{Authors' contributions}

Conceptualization was achieved by GL, ML, IA, GF, AA, EM, $\mathrm{MB}, \mathrm{RC}$ and GI. Methodology was the responsibility of ML, GL, RC and GI. Resources were acquired by AA, EM, GF and MB. Data curation was carried out by GL, MB, AA and R.C. Writing of the original draft preparation was done by ML, GL and GI; writing, review and editing were the responsibility of ML, GL, IA, GF, AA, MB, EM, RC and GI. Supervision was carried out by GI. All authors read and agreed to the published version of the manuscript.

\section{Ethics approval and consent to participate}

Not applicable.

\section{Patient consent for publication}

Not applicable.

\section{Competing interests}

The authors declare that they have no competing interests.

\section{References}

1. Finkel K, Perazella MA and Cohen E: Acute kidney injury-Incidence, Pathogenesis and Outcomes. In: Onco-Nephrology. 1st edition. Elsevier, Amsterdam, pp270-274, 2019.

2. Bratu OG, Cherciu AI, Bumbu A, Lupu S, Marcu DR, Ionita-Radu F, Manea M, Furau C, Diaconu CC and Mischianu DL: Retroperitoneal tumours-treatment and prognosis of tumour recurrence. Rev Chim 70: 190-194, 2019.

3. Marcu DR, Ionita-Radu F, Iorga LD, Manea M, Socea B, Scarneciu I, Isvoranu G, Costache R, Diaconu CC and Bratu OG: Vascular involvement in primary retroperitoneal tumors. Rev Chim 70: 445-448, 2019.

4. Draghici T, Negreanu L, Bratu OG, Stoian AP, Socea B Neagu TP, Stanescu AM, Manuc D and Diaconu CC: Paraneoplastic syndromes in digestive tumors: A review. Rom Biotechnol Lett 24: 813-819, 2019.

5. Perazella MA: Renal vulnerability to drug toxicity. Clin J Am Soc Nephrol 4: 1275-1283, 2009.

6. Kidney Disease: Improving Global Outcomes (KDIGO) CKD Work Group. KDIGO 2012 Clinical Practice Guideline for Acute Kidney Injury. Kidney Int Suppl 1: 1-138, 2012.

7. Christiansen CF, Johansen MB, Langeberg WJ, Fryzek JP and Sørensen HT: Incidence of acute kidney injury in cancer patients: A Danish population-based cohort study. Eur J Intern Med 22: 399-406, 2011

8. International Agency for Research on Cancer: Europe-Global cancer Observatory. Globoscan 2018. https://gco.iarc.fr/today/ data/factsheets/populations/908-europe-fact-sheets.pdf. Accessed October 28, 2020.

9. Bratu OG, Diaconu CC, Mischianu DLD, Constantin T, Stanescu AM, Bungau SG, Ionita-Radu F and Marcu RD Therapeutic options in patients with biochemical recurrence after radical prostatectomy. Exp Ther Med 18: 5021-5025, 2019.

10. Bratu O, Spînu D, Oprea I, Popescu R, Marcu D, Farcaș C, Dinu $M$ and Mischianu D: Complications of radical retropubicprostatectomy-our experience. Romanian J Military Med 118: 23-25, 2015

11. Marcu D, Spinu D, Mischianu D, Socea B, Oprea I and Bratu OG Oncological follow-up after radical prostatectomy. Romanian J Military Med 120: 39-42, 2017.
12. Tataru AL, Furau G, Afilon J, Ionescu C, Dimitriu M, Bratu OG, Tit DM, Bungau A and Furau C: The situation of cervical cancers in the context of female genital cancer clustering and burden of disease in Arad County. Romania J Clin Med 8: 96, 2019.

13. Lameire N, Van Biesen W and Vanholder R: Acute renal failure. Lancet 365: 417-430, 2005.

14. Finkel KW and Kala J: Renal replacement therapy in critically Ill cancer patients. In: Oncologic Critical Care. Nates J and Price K (eds). Springer, Cham, pp1-12, 2019.

15. Daugirdas JT: Physiologic principles and urea kinetic modeling. In: Handbook of Dialysis. 5th edition. Daugirdas JT, Blake PG and Ing TS (eds). Wolters Kluwer, Philadelphia, pp34-65, 2015

16. Fathima N, Kashif T, Janapala RN, Jayaraj JS and Qaseem A: Single-best choice between intermittent vs. continuous renal replacement therapy: A review. Cureus 11: e5558, 2019.

17. PIRRT may help you improve resource utilization, patient care, and early patient mobilization programs. https://www.nxstage. $\mathrm{com} / \mathrm{hcp} / \mathrm{therapies/pirrt/.} \mathrm{Accessed} \mathrm{September} \mathrm{14,} 2020$.

18. Sherman RA, Daugirdas JT and Ing TS: Complications during hemodialysis. In: Handbook of Dialysis. 5th edition. Daugirdas JT, Blake PG and Ing TS (eds). Wolters Kluwer, Philadelphia, pp215-236, 2015

19. Trinh-Trang-Tan MM, Cartron JP and Bankir L: Molecular basis for the dialysis disequilibrium syndrome: Altered aquaporin and urea transporter expression in the brain. Nephrol Dial Transplant 20: 1984-1988, 2005.

20. Mistry K: Dialysis disequilibrium syndrome prevention and management. Int J Nephrol Renovasc Dis 12: 69-77, 2019.

21. Finkel KW and Waguespack DR: Renal replacemet therapies. In: Onco-Nephrology. 1st edition. Finkel K, Perazella MA and Cohen E (eds). Elsevier, Amsterdam, pp290-298, 2019.

22. Fliser T and Kielstein JT: Technology insight: Treatment of renal failure in the intensive care unit with extended dialysis. Nat Clin Pract Nephrol 2: 32-39, 2006.

23. Berbece AN and Richardson RMA: Sustained low-efficiency dialysis in the ICU: Cost, anticoagulation, and solute removal. Kidney Int 70: 963-968, 2006.

24. Baldwin I, Naka T, Koch B, Fealy N and Bellomo R: A pilot randomized controlled comparison of continuous venovenous hemofiltration and extended daily dialysis with filtration: Effect on small solutes and acid-base balance. Intensive Care Med 33: 830-835, 2007.

25. Kielstein JT, Kretschmer U, Ernst T, Hafer C, Bahr MJ, Haller H and Fliser D: Efficacy and cardiovascular tolerability of extended dialysis in critically ill patients: A randomized controlled study. Am J Kidney Dis 43: 342-349, 2004.

26. Schwenger V, Weigand MA, Hoffmann O, Dikow R, Kihm LP, Seckinger J, Miftari N, Schaier M, Hofer S, Haar C, et al: Sustained low efficiency dialysis using a single-pass batch system in acute kidney injury-a randomized interventional trial: The REnal replacement therapy study in intensive care unit patiEnts. Crit Care 16: R140, 2012

27. Cullis B, Abdelraheem M, Abrahams G, Balbi A, Cruz DN, Frishberg Y, Koch V, McCulloch M, Numanoglu A, Nourse P, et al: Peritoneal dialysis for acute kidney injury. Perit Dial Int 34: 494-517, 2014.

28. Schiffl H, Lang SM and Fischer R: Daily hemodialysis and the outcome of acute renal failure. N Engl J Med 346: 305-310, 2002.

29. Ronco C, Bellomo R, Homel P, Brendolan A, Dan M, Piccinni P and La Greca G: Effects of different doses in continuous veno-venous haemofiltration on outcomes of acute renal failure: A prospective randomised trial. Lancet 356: 26-30, 2000.

30. Uchino S: The epidemiology of acute renal failure in the world. Curr Opin Crit Care 12: 538-543, 2006.

31. Kellum JA, Angus DC, Johnson JP, Leblanc M, Griffin M, Ramakrishnan N and Linde-Zwirble WT: Continuous versus intermittent renal replacement therapy: A meta-analysis. Intensive Care Med 28: 29-37, 2002.

32. Tonelli M, Manns B and Feller-Kopman D: Acute renal failure in the intensive care unit: A systematic review of the impact of dialytic modality on mortality and renal recovery. Am J Kidney Dis 40: 875-885, 2002.

33. Sandy D, Moreno L, Lee J and Paganini EP: A randomized stratified, dose equivalent comparison of continuous veno-venous hemodialysis (CVVHD) vs intermittent hemodialysis (IHD) support in ICU acute renal failure patients (ARF). J Am Soc Nephrol 9: 225A, 1998.

34. John S, Griesbach D, Baumgartel M, Weihprecht H, Schmieder RE and Geiger H: Effects of continuous haemofiltration vs intermittent haemodialysis on systemic haemodynamics and splanchnic regional perfusion in septic shock patients: A prospective, randomized clinical trial. Nephrol Dial Transplant 16: 320-327, 2001. 
35. Mehta RL, McDonald BR, Gabbai FB, Pahl M, Pascual MT, Farkas A and Kaplan RM; Collaborative Group for Treatment of ARF in the ICU: A randomized clinical trial of continuous vs intermittent dialysis for acute renal failure. Kidney Int 60:1154-1163, 2001.

36. Uehlinger DE, Jakob SM, Eichelberger M, et al: A randomized, controlled single center study for the comparison of continuous renal replacement therapy with intermittent hemodialysis in critically ill patients with acute renal failure. J Am Soc Nephrol 12: 278A, 2001.

37. Leaf DE and Waika SS: IDEAL-ICU in context. Clin J Am Soc Nephrol 14: 1264-1267, 2019.

38. Zarbock A, Kellum JA, Schmidt C, Van Aken H, Wempe C, Pavenstädt H, Boanta A, Gerß J and Meersch M: Effect of early vs delayed initiation of renal replacement therapy on mortality in critically ill patients with acute kidney injury: The ELAIN randomized clinical trial. JAMA 315: 2190-2199, 2016.

39. Gaudry S, Hajage D, Schortgen F, Martin-Lefevre L, Pons B, Boulet E, Boyer A, Chevrel G, Lerolle N, Carpentier D, et al: for the AKIKI study group: initiation strategies for renal-replacement therapy in the intensive care unit. N Eng J Med 375: 122-133, 2016.

40. Teo BW, Messer JS, Chua HR, How P and Demirjian S: Continuous Renal Replacement Therapies. In: Handbook of Dialysis. 5th edition. Daugirdas JT, Blake PG and Ing TS (eds). Wolters Kluwer, Philadelphia, pp268-304, 2015.

41. Rastegar M, Kitchlu A and Shirali AC: Tumor lysis syndrome In: Onco-Nephrology. 1st edition. Daugirdas JT, Blake PG and Ing TS (eds). Elsevier, Amsterdam, pp275-280, 2019.

42. Kiprov DD, Sanchez A and Pusey C: Therapeutic apheresis. In: Handbook of Dialysis. 5th edition. Daugirdas JT, Blake PG and Ing TS (eds). Wolters Kluwer, Philadelphia, pp333-359, 2015

43. Favà A, Fulladosa X, Montero N, Draibe J, Torras J, Gomà M and Cruzado JM: Treatment of multiple myeloma with renal involvment: the nephrologist's view. CKD 11: 777-785, 2018.

44. Hutchison CA, Cockwell P, Stringer S, Bradwell A, Cook M, Gertz MA, Dispenzieri A, Winters JL, Kumar S, Rajkumar SV, et al: Early reduction of serum-free light chains associates with renal recovery in myeloma kidney. J Am Soc Nephrol 22: 1129-1136, 2011

45. Clark WF, Stewart AK, Rock GA, Sternbach M, Sutton DM, Barrett BJ, Heidenheim AP, Garg AX and Churchill DN; Canadian Apheresis Group: Plasma exchange when myeloma presents as acute renal failure: A randomized, controlled trial. Ann Intern Med 143: 777-784, 2005.

46. Gondouin B and Hutchison CA: High cut-off dialysis membranes: Current uses and future potential. Adv Chronic Kidney Dis 18: 180-187, 2011

47. Hutchison CA, Heyne N, Airia P, Schindler R, Zickler D, Cook M, Cockwell P and Grima D: Immunoglobulin free light chain levels and recovery from myeloma kidney on treatment with chemotherapy and high cut-off haemodialysis. Nephrol Dial Transplant 27: 3823-3828, 2012.
48. Finkel K and Fabbrini P: Paolo fabbrini. High cut-off hemodialysis for myeloma cast nephropathy-do we finally have an answer? J Onco-Nephrol 1: 67-70, 2017.

49. Hutchison CA, Cook M, Heyne N, Weisel K, Billingham L, Bradwell A and Cockwell P: European trial of free light chain removal by extended haemodialysis in cast nephropathy (EuLITE): A randomised control trial. Trials 9: 55, 2008.

50. Bridoux F, Carron PL, Pegourie B, Alamartine E, Augeul-Meunier K, Karras A, Joly B, Peraldi MN, Arnulf B, Vigneau C, et al: Effect of high-cutoff hemodialysis vs conventional hemodialysis on hemodialysis independence among patients with myeloma cast nephropathy: A randomized clinical trial. JAMA 318: 2099-2110, 2017.

51. Vallée AG, Chenine L, Leray-Moragues H, Patrier L, Cognot C, Cartron G, Cristol JP and Canaud B: Online high-efficiency haemodiafiltration achieves higher serum free light chain removal than high-flux haemodialysis in multiple myeloma patients: Preliminary quantitative study. Nephrol Dial Transplant 26: 3627-3633, 2011.

52. Kirsch AH, Lyko R, Nilsson LG, Beck W, Amdahl M, Lechner P, Schneider A, Wanner C, Rosenkranz AR and Krieter DH. Performance of hemodialysis with novel medium cut-off dialyzers. Nephrol Dial Transplant 1: 165-172, 2016.

53. Zweigart C,Boschetti-de-Fierro A,Hulko M,Nilsson LG,Beck W, Storr M and Krause B: Medium cut-off membranes-closer to the natural kidney removal function. Int J Artif Organs 40: 328-334, 2017.

54. Krishnasamy R, Hawley CM, Jardine MJ, Roberts MA, Cho YJ, Wong MG, Heath A, Nelson CL, Sen A, Mount PF, et al: Design and methods of the REMOVAL-HD study: A tRial evaluating Mid cut-Off value membrane clearance of albumin and light chains in haemoDialysis patients. BMC Nephrol 19: 89, 2018

55. Ronco C, Marchionna N, Brendolan A, Neri M, Lorenzin A and Rueda AJ: Expanded haemodialysis: Expanded haemodialysis: From operational mechanism to clinical results. Nephrol Dial Transplant 33 (Suppl 3): iii41-iii47, 2018.

56. Pérez-García $R$ and Alcázar R: The dialyser in the year 2017: Much more than a membrane. Nefrologia 38: 4-7, 2018 (In English, Spanish). 